\title{
Fertility-Related Quality of Life, Gonadal Function, and Erectile Dysfunction in Male Partners of Couples with Unexplained Infertility
}

R. Matthew Coward, MD, FACS ${ }^{a, b}$, Christy Stetter, BS ${ }^{c}$, Allen Kunselman, MA ${ }^{c}$, JC Trussell, MD $^{d}$, Mark C. Lindgren, MD $^{e}$, Ruben R. Alvero, MD $^{f}$, Peter Casson, MD ${ }^{g}$, Gregory M. Christman, MD $^{h}$, Christos Coutifaris, MD, PhDi, Michael P. Diamond, MDj, Karl R. Hansen, MD, PhD ${ }^{k}$, Stephen A. Krawetz, PhD', Richard S. Legro, MD ${ }^{m}$, Randal D. Robinson, MD $^{n}$, James F. Smith, MD, MS $^{\circ}$, Anne Z. Steiner, MD, MPH ${ }^{p}$, Robert A. Wild, MD, PhD $^{k}$, Heping Zhang, PhD $^{q}$, Nanette Santoro, MD $^{r}$, Reproductive Medicine Network aDepartment of Urology, University of North Carolina School of Medicine, Chapel Hill, NC bUNC Fertility, Raleigh, NC

'Department of Public Health Sciences, Penn State College of Medicine, Hershey, PA dDepartment of Urology, Upstate University Hospital, Syracuse, NY

eDepartment of Urology, University of Oklahoma Health Sciences Center, Oklahoma City, OK fDepartment of Obstetrics and Gynecology, Warren Alpert School of Medicine at Brown University, Providence, RI

gNortheastern Reproductive Medicine, Colchester, VT

hDepartment of Obstetrics and Gynecology, University of Florida, Gainesville, FL

'Department of Obstetrics and Gynecology, Perelman School of Medicine, University of Pennsylvania, Philadelphia, PA

jDepartment of Obstetrics and Gynecology, Augusta University, Augusta, GA

kDepartment of Obstetrics and Gynecology, University of Oklahoma Health Sciences Center, Oklahoma City, OK

'Department of Obstetrics and Gynecology, Center for Molecular Medicine and Genetics, Wayne State University School of Medicine, Detroit, MI

mDepartment of Obstetrics and Gynecology, Pennsylvania State University, Hershey, PA nDepartment of Obstetrics and Gynecology, University of Texas Health Science Center at San Antonio, San Antonio, TX

Corresponding Author: R. Matthew Coward, MD FACS, 2113 Physician's Office Building, CB\#7235, 170 Manning Drive, Chapel Hill, NC 27599-7235, Phone: 919-966-8217, Fax: 919-966-0098, mcoward@ med.unc.edu.

Date of first patient's enrollment: August 2, 2010

Trial registration number:Assessment of Multiple Intrauterine Gestations in Ovulation Stimulation (AMIGOS), , clinicaltrials.gov. Trial registration date:January 7, 2010 
'Department of Urology, University of California, San Francisco, San Francisco, CA

PDepartment of Obstetrics and Gynecology, Duke University, Durham, NC

qDepartment of Biostatistics, Yale University School of Public Health, New Haven, CT

'Department of Obstetrics and Gynecology, University of Colorado School of Medicine, Aurora, CO

\section{Abstract}

Purpose: We sought to determine whether lower fertility-related QOL or depression in men of couples with unexplained infertility is associated with low total testosterone (TT) levels, abnormal semen quality, or erectile dysfunction (ED).

Materials and Methods: This study is a secondary analysis of a large, multicentered, randomized controlled trial for couples with unexplained infertility. Male partners had a baseline semen analysis, fasting TT and gonadotropin levels, and completed the Fertility-Related QOL (FertiQOL), Patient Health Questionnaire 9 (PHQ-9), and International Index of Erectile Function (IIEF) surveys. The primary outcomes were TT (low TT defined as $<264 \mathrm{ng} / \mathrm{dL}$ ), semen parameters, and IIEF score. Multivariable logistic regression analyses adjusted for age, race, BMI, education, smoking, alcohol use, length of infertility, and comorbidity.

Results: 708 men (mean age $34.2 \pm 5.6$ ) were included. Fifty-nine (8.3\%) men had a PHQ-9 score ( 25$)$ consistent with depression, 99 (14.0\%) had low TT, and $63(9.0 \%)$ had mild ED or worse. Neither FertiQOL score nor depression was associated with TT or any semen parameter. FertiQOL was inversely associated with ED [adjusted odds ratio (AOR) 1.30; 95\% confidence interval (CI): 1.16, 1.46, for every 5-point decline in score], and depressed men were significantly more likely to have ED than non-depressed men (AOR 6.31; 95\% CI: 3.12, 12.77).

Conclusions: For men in couples with unexplained infertility, both lower fertility-related QOL and depression are strongly associated with ED, but neither is associated with spermatogenesis or testosterone levels. ED in infertile men merits longitudinal investigation in future studies.

\section{Keywords}

Erectile dysfunction; Hypogonadism; Infertility; Infertility; Male; Quality of life

\section{Introduction}

The impact of infertility on the quality of life (QOL) of men can have far-reaching effects. Men in infertile couples report a greater sense of loss and stigma, more negative emotion, and a lower QOL when the diagnosis is male factor infertility as opposed to unexplained or female factor infertility ${ }^{1-3}$. Lower QOL and associated stress can negatively affect a man's fertility via the two primary male gonadal functions of spermatogenesis and testosterone production, the latter of which is critical for male sexual function including erectile function ${ }^{4}$. Both perceived stress and actual stressful life events have been associated with lower semen quality within the general male population ${ }^{5}$. In addition, during periods of acute stress, testosterone may also become suppressed ${ }^{6}$. Both semen quality and testosterone 
levels have been found to be reduced in infertile men reporting higher psychological stress compared to infertile men reporting lower stress ${ }^{7}$.

The Fertility-Related QOL (FertiQOL) survey is a 34-item behavioral instrument specifically designed to assess the burden of infertility in men and women who are experiencing fertility problems ${ }^{8}$. The FertiQOL assesses emotional, mind/body, relational, and social core domains, as well as a treatment domain and two additional items that measure overall satisfaction with physical health and QOL. The survey is scored $0-100$, with higher scores indicating better QOL. A recent publication examining FertiQOL scores in addition to demographic and morphometric data of couples who participated in two large clinical trials found that younger male partners reported decreased QOL. Notably, this study did not report gonadal or sexual function ${ }^{9}$.

The effect of QOL and depression on male gonadal and sexual function has never been studied in a large cohort of men in infertile couples. Therefore, we sought to assess these relationships in a robust sample of well-characterized men within a large, randomized controlled trial for treatment of unexplained infertility. We hypothesized that lower FertiQOL scores in men would be associated with lower total testosterone (TT) levels, abnormal semen parameters, and ED. Additional secondary hypotheses that we tested included the following: 1) Men with PHQ-9 scores indicative of depression ( 25 ) would be more likely to have low TT, abnormal semen parameters, and ED; and, 2) Erectile function would be overall worse in men with lower TT.

\section{Materials and Methods}

This study is a secondary analysis of data from the Assessment of Multiple Intrauterine Gestations from Ovarian Stimulation (AMIGOS) trial, a prospective, randomized, multicentered clinical trial investigating pregnancy, live birth, and multiple pregnancy rates following ovarian stimulation and intrauterine insemination for couples with unexplained infertility. The AMIGOS trial enrolled 900 couples with $>1$ year of unexplained infertility with regular, unprotected intercourse. Eligible female partners were between 18-40 years of age and had regular ovulation, a normal uterine cavity, and at least one patent Fallopian tube. Further details on the study design and inclusion/exclusion criteria of the trial have been previously described ${ }^{10}$, as have the baseline characteristics of the study population and results for the primary outcomes ${ }^{11,12}$. Informed consent was obtained on all participants and the study was IRB approved at all sites.

Eligible male partners for AMIGOS had a semen analysis with $>5$ million total motile sperm assessed within a year of enrollment. Male partners completed the FertiQOL, the Patient Health Questionnaire 9 (PHQ-9) to assess depression, and the International Index of Erectile Function (IIEF) surveys at baseline. The treatment domain of the FertiQOL instrument was not assessed because the survey was administered prior to treatment. Upon enrollment, baseline TT, luteinizing hormone (LH), and follicle-stimulating hormone (FSH) levels were measured, generally in the morning as the patients were fasting, although an exact time was not mandated or recorded. The University of Virginia Ligand Assay and Analysis Core Laboratories processed all of the serum samples, and the TT assay utilized was a 
radioimmunoassay from Siemens Diagnostics (Los Angeles, CA). The assay quality control measures included a sensitivity of $10 \mathrm{ng} / \mathrm{dL}$, a range of $10-1,000 \mathrm{ng} / \mathrm{dL}$, an intra-assay coefficient of variation of $4.0 \%$, and an inter-assay coefficient of variation of $7.1 \%$.

Low TT was defined as $<264 \mathrm{ng} / \mathrm{dL}$ based on recently published data utilizing a harmonized normal range from four different large cohorts of healthy European and American men 1939 years of age which identified $264 \mathrm{ng} / \mathrm{dL}$ as the $2.5^{\text {th }}$ percentile $^{13}$. Semen parameters were assessed from the screening semen analysis and included semen volume, sperm concentration, percentage of total motility, and Kruger strict morphology. Each semen parameter was dichotomized as normal or abnormal by the accepted World Health Organization (2010) $5^{\text {th }}$ Edition's criteria for lower limit of normal, therefore abnormal cutoffs were established with a semen volume of $<1.5 \mathrm{~mL}$, sperm concentration $<15$ $\times 10^{6} / \mathrm{mL}$, total sperm motility $<40 \%$, and strict morphology $<4 \%$. Erectile function was dichotomized as normal $(\geq 26)$ or as mild ED or worse $(<26)$ by the Erectile Function Domain of the IIEF survey (IIEF-EF). Depression was dichotomized into not depressed $(<5)$ versus mild depression or worse ( $\geq 5$ ) on the PHQ-9 survey.

Age, BMI, and FertiQOL score are reported as mean \pm standard deviation. TT, LH levels, FSH levels, and semen parameters are reported as median with interquartile range.

Multivariable logistic regression models were used to assess the association between FertiQOL scores and TT levels, semen parameters, and erectile function. In addition, multivariable logistic regression was used to also analyze these same outcomes as a function of depression. All regression models were adjusted for age, race, BMI, education, smoking, alcohol use, length of infertility, and comorbidity defined as having any of the following three conditions: hypertension, diabetes, and/or heart disease. For logistic regression, the magnitude of the association is reported as an adjusted odds ratio (AOR) and 95\% confidence interval (CI). All hypothesis tests were two-sided, and a p-value $<0.05$ was considered significant. All analyses were performed using SAS software version 9.4 (SAS Institute Inc., Cary, NC).

\section{Results}

Of the 900 couples enrolled in the AMIGOS trial, 739 men had complete data for FertiQOL and PHQ-9 scores, TT levels, and baseline semen parameters. We excluded 31 men with incomplete covariate data (age, race, BMI, education, smoking, alcohol use, length of infertility, and comorbidity status), resulting in a total of 708 men with a mean age of 34.2 \pm 5.6 years, included in the study. The patient demographics and survey data are included in Table 1 . The cohort was primarily comprised of healthy, non-smoking, Caucasian men with an education beyond high school. There were 59 (8.3\%) men who had a PHQ-9 score consistent with mild depression or worse ( 25$)$. Sixty-three men $(9.0 \%)$ had mild ED or worse (IIEF-EF <26).

Gonadal function was measured by TT levels and semen parameters. The median TT level for the cohort was $413 \mathrm{ng} / \mathrm{dL}$ ( $318-525 \mathrm{ng} / \mathrm{dL})$. There were 99 (14.0\%) men with low TT ( $<264 \mathrm{ng} / \mathrm{dL}$ ). The median LH level was $3.1 \mathrm{mIU} / \mathrm{mL}(2.3-4.5 \mathrm{mIU} / \mathrm{mL})$, and the median FSH was $3.8 \mathrm{mIU} / \mathrm{mL}(2.8-5.4 \mathrm{mIU} / \mathrm{mL})$. The median semen parameters were all within 
normal limits by the 2010 World Health Organization Manual, $5^{\text {th }}$ Edition. The median semen volume was $2.9 \mathrm{~mL}(2.0-3.7 \mathrm{~mL})$, sperm concentration was $48.0 \times 10^{6} / \mathrm{mL}(27.0-$ $\left.85.5 \times 10^{6} / \mathrm{mL}\right)$, total sperm motility was $57.0 \%(50.0-65.0 \%)$, and Kruger strict morphology was $9.0 \%(5.0-18.0 \%)$. There were $84(11.9 \%)$ men with low semen volume, $69(9.8 \%)$ with low sperm concentration, 88 (12.4\%) with low motility, and $116(19.0 \%)$ with low morphology.

The multivariable logistic regression analyses are presented in Table 2 and 3. Neither FertiQOL score nor depression was associated with TT or any semen parameter. FertiQOL score was inversely associated with risk of ED. On average, every 5-point decrease in the FertiQOL score resulted in a 30\% increase in the odds of having mild ED or worse (AOR 1.30; 95\% CI: 1.16, 1.46) (Table 2). Depressed men were more likely to have mild ED or worse compared with non-depressed men (AOR 6.31; 95\% CI: 3.12, 12.77) (Table 3). There was no association between low TT and ED in the cohort (AOR 1.00; 95\% CI: 0.47, 2.13).

\section{Discussion}

In this secondary analysis of data from a large, prospective, multicentered randomized controlled trial of couples with unexplained infertility, we found that both lower fertilityrelated QOL and depression in the male partners were strongly associated with ED. We demonstrated that neither lower fertility-related QOL nor depression were associated with low TT or abnormal semen parameters in the entire cohort.

A link between QOL and fertility is supported by evidence of biological cross-talk between the hypothalamic-pituitary-adrenal and hypothalamic-pituitary-gonadal axes ${ }^{14}$. Results from a recent meta-analysis suggest that interventions aimed at reducing psychosocial stress in couples undergoing assisted reproduction may improve clinical pregnancy rates ${ }^{15}$. Most previous studies have focused on female infertility, with only a few addressing the men of infertile couples and the role of QOL and depression on these men's gonadal and sexual function. Within the general population, reduced semen quality may be present in men with self-reported high stress, although testosterone levels may remain normal in these, otherwise healthy, men ${ }^{16}$. When present, testosterone deficiency has been independently associated with reduced health-related QOL in a variety of populations. In addition to men with $\mathrm{ED}^{17}$, diabetes ${ }^{18}$, and other medical co-morbidities, it is well known that health-related QOL is closely correlated with testosterone levels even among community-dwelling men ${ }^{19}$. Young men with evidence of biochemical hypogonadism are more likely to have an impaired emotional state, lower QOL, and lower cognitive function compared with age-matched, healthy controls ${ }^{20}$.

The relationship between depression and ED is well established. Depression affects approximately one in six people ${ }^{21}$. Data from the landmark Massachusetts Male Aging Study demonstrated that, among healthy men aged 40 to 70 years, those with depression were over $80 \%$ more likely to have moderate ED or worse compared to men without depression (AOR 1.82; 95\% CI: 1.21, 2.73) ${ }^{22}$. The present study found that healthy male partners in infertile couples with symptoms of depression have a much greater risk of ED well above that of community dwelling men. We have shown that men with depression in 
infertile couples were over six times more likely to have mild ED or worse than their nondepressed counterparts (AOR 6.31; 95\% CI: 3.12, 12.77). Because a bidirectional relationship may exist between depression, $\mathrm{ED}$, and gonadal function, after our primary analysis was completed, we additionally explored the reverse relationships, testing the outcome of depression as a function of ED and low TT. The multivariable logistic regression result for the reverse was indeed similar to those reported in Table 3, as men in infertile couples with ED were over six times more likely to have depression than men with normal erectile function (AOR 6.09; 95\% CI: 3.02, 12.31). Low TT did not predict depression (AOR $0.50 ; 95 \%$ CI: $0.20,1.26)$.

We did not identify a relationship between ED and lower TT levels in this cohort of men with unexplained infertility. ED is a well-recognized symptom of clinical hypogonadism ${ }^{23}$. In the present study, a clinical exam and symptom assessment were not performed for the study participants, and therefore it is important to recognize that the low TT levels assessed in this study represent a biochemical finding without a clinical correlate. Additionally, enrollment criteria included having regular, unprotected intercourse and a normal semen analysis, which may have excluded men with a TT low enough to reduce their semen parameters below the eligibility threshold for this study. Therefore, while $14.0 \%$ of this group of men in infertile couples had biochemical evidence of low TT on a single determination, the cohort was generally healthy, lacking significant baseline sexual dysfunction. These limitations of the parent trial, including the health status and sexual function for male partners required for eligibility in this study sample, may be why a relationship between low TT levels and ED was not demonstrated.

This study is also limited by the methodology of data collection for the AMIGOS trial with a single baseline laboratory assessment while fasting but not definitively drawn in the morning. This provides only one cross-sectional evaluation of the men in the study prior to initiation of treatment. The diagnosis of clinical hypogonadism is recommended to be made by two, separate lab draws in the morning hours ${ }^{24,25}$. In addition, a complete history and physical exam along with evaluation for the symptoms of hypogonadism were not performed, therefore symptomatic hypogonadism as a clinical diagnosis was not obtained. Without a complete medical history including medication list, we were unable to assess for prior history of clinical depression or use of anti-depressants, prior history of ED or use of phosphodiesterase type 5 inhibitors, or prior history of hypogonadism or use or hormone therapy. Although the sample size was quite large $(n=708)$ and likely the prevalence of such pre-existing conditions is low, the lack of this history could have skewed our results. Despite these limitations, this sizeable cohort of healthy men was studied in a prospective, randomized, multicentered clinical trial and provides a unique and well-characterized perspective into the baseline fertility-related QOL, gonadal, and erectile function in male partners of couples with unexplained infertility.

\section{Conclusions}

For men in couples with unexplained infertility, neither fertility-related QOL nor depression was associated with gonadal dysfunction as measured by a single TT level and semen analysis. Both lower fertility-related QOL and depression among male partners in couples 
with unexplained infertility were strongly associated with ED, implying that men in infertile couples should be evaluated for depression if ED is present, and vice versa. ED in infertile men merits dedicated investigation in future studies.

\section{Acknowledgements}

The authors recognize the invaluable contributions of Dr. Esther Eisenberg, MD, MPH, Project Scientist for the Reproductive Medicine Network.

Source of Funding: Supported by grants from the National Institutes of Health, the Eunice Kennedy Shriver National Institute of Child Health and Human Development (U10 HD077844, U10 HD077680, U10 HD39005, U10 HD38992, U10 HD27049, U10 HD38998, U10 HD055942, U10 HD055944, U10 HD055936, and U10 HD055925, to the University of Virginia Center for Research in Reproduction Ligand Assay and Analysis Core of the Specialized Cooperative Centers Program in Reproduction and Infertility Research), the National Center for Research Resources, the National Center for Advancing Translational Sciences (UL1 TR000127, to Pennsylvania State University), and by the American Recovery and Reinvestment Act.

\section{References}

1. Abbey A, Andrews FM, Halman LJ: Provision and receipt of social support and disregard: what is their impact on the marital life quality of infertile and fertile couples? J Pers Soc Psychol, 68: 455, 1995 [PubMed: 7714725]

2. Nachtigall RD, Becker G, Wozny M: The effects of gender-specific diagnosis on men's and women's response to infertility. Fertil Steril, 57: 113, 1992 [PubMed: 1730303]

3. Smith JF, Walsh TJ, Shindel AW et al.: Sexual, marital, and social impact of a man's perceived infertility diagnosis. J Sex Med, 6: 2505, 2009 [PubMed: 19619144]

4. Schneid-Kofman N, Sheiner E: Does stress effect male infertility?--a debate. Med Sci Monit, 11: SR11, 2005 [PubMed: 16049396]

5. Janevic T, Kahn LG, Landsbergis P et al.: Effects of work and life stress on semen quality. Fertil Steril, 102: 530, 2014 [PubMed: 24856463]

6. Lieberman HR, Farina EK, Caldwell J et al.: Cognitive function, stress hormones, heart rate and nutritional status during simulated captivity in military survival training. Physiol Behav, 165: 86, 2016 [PubMed: 27374427]

7. Bhongade MB, Prasad S, Jiloha RC et al.: Effect of psychological stress on fertility hormones and seminal quality in male partners of infertile couples. Andrologia, 47: 336, 2015 [PubMed: 24673246]

8. Boivin J, Takefman J, Braverman A: The Fertility Quality of Life (FertiQoL) tool: development and general psychometric properties. Fertil Steril, 96: 409, 2011 [PubMed: 21458806]

9. Santoro N, Eisenberg E, Trussell JC et al.: Fertility-related quality of life from two RCT cohorts with infertility: unexplained infertility and polycystic ovary syndrome. Hum Reprod, 31: 2268, 2016 [PubMed: 27402910]

10. Diamond MP, Mitwally M, Casper R et al.: Estimating rates of multiple gestation pregnancies: sample size calculation from the assessment of multiple intrauterine gestations from ovarian stimulation (AMIGOS) trial. Contemp Clin Trials, 32: 902, 2011 [PubMed: 21787883]

11. Diamond MP, Legro RS, Coutifaris C et al.: Letrozole, Gonadotropin, or Clomiphene for Unexplained Infertility. N Engl J Med, 373: 1230, 2015 [PubMed: 26398071]

12. Diamond MP, Legro RS, Coutifaris $C$ et al.: Assessment of multiple intrauterine gestations from ovarian stimulation (AMIGOS) trial: baseline characteristics. Fertil Steril, 103: 962, 2015 [PubMed: 25707331]

13. Travison TG, Vesper HW, Orwoll E et al.: Harmonized Reference Ranges for Circulating Testosterone Levels in Men of Four Cohort Studies in the United States and Europe. J Clin Endocrinol Metab, 102: 1161, 2017 [PubMed: 28324103]

14. Viau V: Functional cross-talk between the hypothalamic-pituitary-gonadal and -adrenal axes. J Neuroendocrinol, 14: 506, 2002 [PubMed: 12047726] 
15. Frederiksen Y, Farver-Vestergaard I, Skovgard NG et al.: Efficacy of psychosocial interventions for psychological and pregnancy outcomes in infertile women and men: a systematic review and metaanalysis. BMJ Open, 5: e006592, 2015

16. Nordkap L, Jensen TK, Hansen AM et al.: Psychological stress and testicular function: a crosssectional study of 1,215 Danish men. Fertil Steril, 105: 174, 2016 [PubMed: 26477499]

17. Hwang TI, Lo HC, Tsai TF et al.: Association among hypogonadism, quality of life and erectile dysfunction in middle-aged and aged male in Taiwan. Int J Impot Res, 19: 69, 2007 [PubMed: 16688209]

18. Brooke JC, Walter DJ, Kapoor D et al.: Testosterone deficiency and severity of erectile dysfunction are independently associated with reduced quality of life in men with type 2 diabetes. Andrology, 2: 205, 2014 [PubMed: 24574152]

19. Hsu B, Cumming RG, Blyth FM et al.: Longitudinal and cross-sectional relationships of circulating reproductive hormone levels to self-rated health and health-related quality of life in communitydwelling older men. J Clin Endocrinol Metab, 99: 1638, 2014 [PubMed: 24552220]

20. Lasaite L, Ceponis J, Preiksa RT et al.: Impaired emotional state, quality of life and cognitive functions in young hypogonadal men. Andrologia, 46: 1107, 2014 [PubMed: 24313565]

21. Kessler RC, Berglund P, Demler O et al.: The epidemiology of major depressive disorder: results from the National Comorbidity Survey Replication (NCS-R). JAMA, 289: 3095, 2003 [PubMed: 12813115]

22. Araujo AB, Durante R, Feldman HA et al.: The relationship between depressive symptoms and male erectile dysfunction: cross-sectional results from the Massachusetts Male Aging Study. Psychosom Med, 60: 458, 1998 [PubMed: 9710291]

23. Traish AM, Miner MM, Morgentaler A et al.: Testosterone deficiency. Am J Med, 124: 578, 2011 [PubMed: 21683825]

24. Khera M, Adaikan G, Buvat J et al.: Diagnosis and Treatment of Testosterone Deficiency: Recommendations From the Fourth International Consultation for Sexual Medicine (ICSM 2015). J Sex Med, 13: 1787, 2016 [PubMed: 27914560]

25. Mulhall JP, Trost LW, Brannigan RE et al.: Evaluation and Management of Testosterone Deficiency: AUA Guideline. J Urol, 2018 
Table 1.

Baseline Patient Demographics and Survey Data for the Male Cohort ( $\mathrm{n}=708$ )

\begin{tabular}{|l|l|}
\hline & $\mathbf{n}(\boldsymbol{\%})$ or mean \pm SD \\
\hline Age (years) & $34.2 \pm 5.6$ \\
\hline BMI $\left(\mathrm{kg} / \mathrm{m}^{2}\right)$ & $28.8 \pm 5.6$ \\
\hline Race: & \\
\hline Caucasian & $592(83.6)$ \\
\hline African American & $54(7.6)$ \\
\hline Other & $62(8.8)$ \\
\hline Education beyond high school & $588(83.1)$ \\
\hline Current Smoker & $89(12.6)$ \\
\hline Current Alcohol Use & $577(81.5)$ \\
\hline Length of infertility (months) & $34.2 \pm 24.2$ \\
\hline Comorbidities: & \\
\hline Diabetes & $25(3.5)$ \\
\hline Hypertension & $78(11.0)$ \\
\hline Heart disease & $24(3.4)$ \\
\hline FertiQOL Score & $83.1 \pm 10.9$ \\
\hline PHQ-9 Score & $1.5 \pm 2.6$ \\
\hline IIEF-EF Score & $28.7 \pm 3.5$ \\
\hline ED Severity (by IIEF-EF Score): & \\
\hline Normal (26-30) & $636(89.8)$ \\
\hline Mild (22-25) & $37(5.2)$ \\
\hline Mild to moderate (17-21) & $12(1.7)$ \\
\hline Moderate (score 11-16) & $8(1.1)$ \\
\hline Severe (score 1-10) & $6(0.9)$ \\
\hline Missing & $9(1.3)$ \\
\hline
\end{tabular}


Table 2.

Multivariable Logistic Regression Analysis*

\begin{tabular}{|l|l|l|l|}
\hline Predictor & Outcome & $\begin{array}{l}\text { Adjusted Odds } \\
\text { Ratio (95\% CI) }\end{array}$ & p-value \\
\hline FertiQOL Score (per 5-point decrease) & Low Total Testosterone $(<264 \mathrm{ng} / \mathrm{dL})$ & $0.99(0.89,1.09)$ & 0.82 \\
\hline & Low Semen Volume $(<1.5 \mathrm{~mL})$ & $0.99(0.89,1.10)$ & 0.80 \\
\hline & Low Sperm Concentration $(<15106 / \mathrm{mL})$ & $0.92(0.82,1.05)$ & 0.22 \\
\hline & Low Total Sperm Motility $(<40 \%)$ & $1.04(0.94,1.14)$ & 0.50 \\
\hline & Low Kruger Strict Morphology $(<4 \%)$ & $0.97(0.88,1.06)$ & 0.48 \\
\hline & Erectile Dysfunction $($ IIEF-EF <26) & $1.30(1.16,1.46)$ & $<.0001$ \\
\hline Depression (PHQ-9 score 25 vs $<5$ (reference group)) & Low Total Testosterone $(<264 \mathrm{ng} / \mathrm{dL})$ & $0.47(0.18,1.20)$ & 0.11 \\
\hline & Low Semen Volume $(<1.5 \mathrm{~mL})$ & $0.47(0.16,1.36)$ & 0.17 \\
\hline & Low Sperm Concentration $(<15106 / \mathrm{mL})$ & $0.62(0.21,1.79)$ & 0.37 \\
\hline & Low Total Sperm Motility $(<40 \%)$ & $1.17(0.55,2.52)$ & 0.68 \\
\hline & Low Kruger Strict Morphology $(<4 \%)$ & $0.92(0.42,2.02)$ & 0.84 \\
\hline & Erectile Dysfunction $($ IIEF-EF <26) & $6.31(3.12,12.77)$ & $<.0001$ \\
\hline
\end{tabular}

Each model is adjusted for age, race, BMI, education, smoking, alcohol use, length of infertility, and comorbidity. 
Table 3.

Multivariable Logistic Regression Analysis as a Function of Depression *

\begin{tabular}{|l|l|l|l|l|}
\hline & \multicolumn{3}{|c|}{ Depressed (n= 59) vs. Not Depressed (n = 649) } \\
\hline Outcome & $\begin{array}{l}\text { Depressed } \\
\text { (PHQ-9 score } \leq)\end{array}$ & $\begin{array}{l}\text { Not Depressed } \\
\text { PHQ-9 score <5, } \\
\text { reference group) }\end{array}$ & $\begin{array}{l}\text { Adjusted Odds } \\
\text { Ratio (95\% CI) }\end{array}$ & p-value \\
\hline $\begin{array}{l}\text { Low Total Testosterone } \\
(<264 \text { ng/dL) }\end{array}$ & $6 / 59(10.2 \%)$ & $93 / 649(14.3 \%)$ & $0.47(0.18,1.20)$ & 0.11 \\
\hline $\begin{array}{l}\text { Low Semen Volume } \\
(<1.5 \mathrm{~mL})\end{array}$ & $4 / 59(6.8 \%)$ & $80 / 649(12.3 \%)$ & $0.47(0.16,1.36)$ & 0.17 \\
\hline $\begin{array}{l}\text { Low Sperm Concentration } \\
(<15106 / \mathrm{mL})\end{array}$ & $4 / 59(6.8 \%)$ & $65 / 649(10.0 \%)$ & $0.62(0.21,1.79)$ & 0.37 \\
\hline $\begin{array}{l}\text { Low Total Sperm Motility } \\
(<40 \%)\end{array}$ & $9 / 59(15.3 \%)$ & $79 / 649(12.2 \%)$ & $1.17(0.55,2.52)$ & 0.68 \\
\hline $\begin{array}{l}\text { Low Kruger Strict Morphology } \\
(<4 \%)\end{array}$ & $9 / 48(18.8 \%)$ & $107 / 563(19.0 \%)$ & $0.92(0.42,2.02)$ & 0.84 \\
\hline $\begin{array}{l}\text { Erectile Dysfunction } \\
(\text { IIEF-EF <26) }\end{array}$ & $18 / 59(30.5 \%)$ & $45 / 640(7.0 \%)$ & $6.31(3.12,12.77)$ & $<.0001$ \\
\hline
\end{tabular}

Each model is adjusted for age, race, BMI, education, smoking, alcohol use, length of infertility, and comorbidity. 